\title{
Application of Profile Control Technology to increase the Degree of Fire Driving in Multi-Layer Oil Reservoirs
}

\author{
Xu Xin \\ Liaohe Oil Field Company, CNP \\ Northeast Petroleum University
}

Keywords: fire flooding, heavy oil reservoir

\begin{abstract}
Fire flooding technology is an effective alternative for steam production after steam stimulation and steam flooding in heavy oil reservoirs. With the accelerating development process, various contradictions in the development process of fire flooding are becoming increasingly prominent. There are defects mainly in fire-flooding gas injection wells, inspiratory inhomogeneity, inconsistent firing speeds, and gas production. Aiming at the problems existing in the fire flooding test, the chemical control technology of fire flooding and well drilling was studied. The high temperature profile control agent formulation system for fire flooding gas wells and the high temperature sealant formulation system for fire flooding production wells were developed to form a fire flooding injection system with well control and matching technology. Field application shows that this technology can effectively improve the heterogeneity of reservoirs, block the air channel, expand the volume of fire lines, increase the degree of oil layer utilization, and promote the homogeneous advancement of the fire front in the vertical and horizontal planes, thereby improving the overall development of fire flooding and effect.
\end{abstract}

\section{Introduction}

The outflow of oil and gas wells is a common problem in the development of oil fields, especially in oilfields where oil production and water injection are used to develop multi-layer coal mining methods. With the development of oilfields, the continuous advancement of formation water (bearwater, bottom water) or injection water can lead to oil fields due to the heterogeneity of the formation, the difference in oil/water flow ratio, and improper development plans or measures. The single-layered water tongue protrudes, and the water well rises quickly, leading to premature flooding, resulting in a decrease in oil recovery. At present, the most widely used measure for oilfields is the water plugging (for wells) technology, which is achieved by adjusting the production structure of the production layer under the condition that the original well pattern is not changed. The extensive application of production well water plugging technology in the field has effectively improved the volume and volume of injected water, adjusted the structure of the oil reservoir, increased the output and recovery rate, and it is a stable oil control and water control during the development of oilfield water injection. Key technology.

Oil well profile control technology is to inject profile control agent without water, in accordance with the permeability of the production wells of the oil wells, and the natural selection of blocking agent for each layer of water discharge capacity. High production well sections or intervals for high water cut oil wells. Selective deep plugging is carried out, and through over-displacement, the plugging layer is plugged and not dead, so as to increase the swept volume in the wells in the middle far wells, adjust the liquid production profile, and change the purpose of the outlet channel.

\section{Differences between Profile Control Technology for Heavy Oil Wells and Conventional Water Plugging Technology}

Block but not die Heavy oil well profile control technology has used over-displacement technology, so that after the plugging of the water layer still has some seepage channels. The 
conventional water-blocking technology completely blocked the water layer. High aquifers are usually the main stratum or the I strata, and blockage is not conducive to the improvement of oil recovery of these reservoirs. This complete blockage is very detrimental to the improvement of the recovery of the layer. The

The requirements for the matching water-seeking process are not high. The heavy oil well profile control technology mainly depends on the profile control agent to naturally enter the high aquifer under different seepage resistance, and the judgment of the water layer does not need to be very accurate. Under normal circumstances, no water is found. Conventional water-blocking technology must be based on accurate water search, otherwise the efficiency is very low. The

Has a certain role in expanding the sweep coefficient. Because of the deep plugging, plugging and dead process, it is equivalent to placing the profile control agent from the well to the oil well (deep profile control), so that the oil well profile control technology can improve the injection. The wave and volume of water change the seepage field of the well in the wells with high water depth in the oil well, and can play the role of planar adjustment for other oil wells in the same well group. This is difficult to achieve for deep well profile control.

\section{Non-selective Blocking Agent Pumps}

After the non-selective blocking agent is pumped into the formation, the plugging agent is blocked everywhere, ie both the outlet passage and outlet passage must be plugged. The method of plugging water with this plugging agent is called non-selective water plugging. The non-selective water plugging agent can be configured as an aqueous solution on the ground. After being pumped into the formation, it can generate gel, thereby blocking the formation. If the resin solution is pumped into the formation, it will produce insoluble resin solids and has high sealing strength. After the formation is sealed by the resin, it loses its ability to produce water and produce oil. Two-fluid method can also be used to pump the two components into the formation separately to make them collide at a predetermined position to produce a solid precipitate to achieve the purpose of plugging. Solid particles can also be suspended in oil or water, such as oil-based cements, water-based cements, clays, and fly ash. After being pumped into the formation, they solidify into sediments and seal the formation. Non-selective water plugging can be used for special operations. Such as sealing the outer tube, single water layer, bottom water coning and borehole, it can also be used to seal fractured strata or large pores.

\section{Water Injection Wells for Profile Control Technology}

In the oilfields developed for water injection, due to the obvious heterogeneity of the formation and the large difference in permeability, the injected water protrudes along the high-permeability zone, resulting in a rapid increase in the moisture content of the well and a rapid drop in oil production. In order to maintain the stable production of oil fields and improve oil recovery, the water injection well profile adjustment work has been generally carried out at home and abroad, which has made great contributions to the stable production of oil fields and enhanced oil recovery. The advantages of the injection well profile control are: it does not affect the normal production of oil wells, one injection well profile control, multiple wells are effective, construction is convenient, and the effective period is long. The mechanism of the profile adjustment of the injection wells is the diversion effect, the improvement of the fluidity ratio and the physical blockage, which are both the adsorption and the residual resistance. The diversion effect is based on the selective entry mechanism of the formation to the plugging agent. When the plugging agent is pumped into the formation, it preferentially enters the high permeability section and generates the gel, gel material or solid precipitate within a predetermined time, thereby blocking the high. The permeate interval forces the injected water to change its flow pattern and flow to the low permeability interval, thus increasing the volume of the injected water and increasing the efficiency of the oil sweep. As a result, the crude oil in the low permeability interval is displaced and flows to the production well. To increase oil precipitation effect. The improvement of the flow ratio is the selective plugging of 
the polymer sol, or the gel formed by the polymer and the cross-linking agent. In the water injection process, the injected water flows along the edge of the polymer, and the part of the polymer in contact with the water gradually swells and dissolves, increasing the viscosity of the water, changing the flow ratio, increasing the area sweep efficiency, and simultaneously expanding the The profile adjustment radius. The physical plugging effect is due to the fact that many reactive groups on the polymer chain crosslink with the polymer to form a network structure. This structure contains water in the lattice structure to form a viscoelastic jelly, which is a frozen colloid. The formation of physical blockage in the porous medium prevents the flow of water through or changes the direction of the flow of water; molecules that are not cross-linked and polar groups on the molecule can be crimped in the pores and also act to block the flow of water; while on the molecular chain The polar groups are adsorbed on the rock surface, which increases the "stickiness" of these polymer chains and frozen colloids to the rock surface, improves the residual resistance of the profile control to the rock, and enhances the water plugging effect.

\section{Conclusion}

Application of profile modification technology to improve the industrialization of multi-layer oilfield fire-driven oilfields has made breakthroughs and rejuvenated the old oilfield. This technology covers 1 billion tons of heavy oil geological reserves and increases the recoverable reserves of 300 million tons, which has broad application prospects. Under the condition that the surface air compressor performance can be met, the development of ultra-deep heavy oil fire-fighting will have more technical and economic advantages than steam injection. For thin layers and thin interbedded thick oils, steam injection also faces the problems of large heat loss (heat transfer to the lid, bottom layer and interlayer), low thermal efficiency and low economic efficiency. Through certain technological breakthroughs, the use of fire-fighting technology to develop such reservoirs is expected to reduce mining costs and increase economic efficiency.

\section{References}

[1] Wang Yuanji, He Jiangchuan, Liao Guangzhi, Wang Zhengmao, Development History and Application Prospects of Domestic Fire Displacement Technology[J] Journal of Petroleum and Chemistry, Vol.33, No.5, 2012,

[2] Wang Ziyao, Li Zhiping, Zhao Zhihua, Discussion on the application prospects of oil production technology in fire reservoirs [C], Inner Mongolia Petrochemical, 2013

[3] Zhang Jinghua, Yang Shuanghu, Wang Qinglin. Fire flooding [M]. Beijing: Petroleum Industry Press, 2012

[4] Zhang Fangli, Technical Review of Fire Formation, [A] Special Oil and Gas Reservoir, 2013 